\title{
Knotted Solitons in a Charged Two-Condensate Bose System
}

\author{
Yishi Duan, Xinhui Zhang.* Yuxiao Liu, and Li Zhao \\ Institute of Theoretical Physics, Lanzhou University, \\ Lanzhou 730000, People's Republic of China
}

\begin{abstract}
By making use of the decomposition of $U(1)$ gauge potential theory and the $\phi$-mapping method, we propose that a charged two-condensate Bose system possesses vortex lines and two classes of knotted solitons. The topological charges of the vortex lines are characterized by the Hopf indices and the Brower degrees of $\phi$-mapping, and the knotted solitons are described by the nontrivial Hopf invariant and the $\mathrm{BF}$ action, respectively.

PACS numbers: 74.20.De, 11.15.Ex, 03.75.Mn

Keywords: Two-Condensate Bose System, Vortex Lines.
\end{abstract}

*Electronic address: zhangxingh03@st.lzu.edu.cn 


\section{INTRODUCTION}

Multigap superconductivity has been discussed in the late 1950's for materials with a varying strength of electron-phonon interactions between different pieces of the Fermi surface [1, 2]. Since then, great attention has been directed towards understanding the detailed nature of superconductivity in this simple intermetallic compound. In recent years, ab inito calculations [3, 4, 5] have showed that $M g B_{2}$ has two weakly coupled gaps: $\Delta_{\sigma} \approx 7 m e v$ and $\Delta_{\pi} \approx 2.5 \mathrm{mev}$, residing on disconnected sheets of the Fermi surface formed by in-plane $P_{x y}$ boron orbitals (the $\sigma$-bands) and out-of -plane $P_{z}$ boron orbitals (the $\pi$-bands). The most striking consequences of the two gaps are the unusual anisotropic feature of $M g B_{2}$ under magnetic field, for example inequality between the penetration depth and the upper critical field anisotropy, and their variations with temperature [6]. Moreover, the experiment of scanning tunneling spectroscopy (STS) measurements on single crystal $M g B_{2}$ shows that the coherent length in the $\pi$ band is approximately $50 \mathrm{~nm} \mathrm{[7]} \mathrm{which} \mathrm{is} \mathrm{much} \mathrm{larger} \mathrm{than}$ an estimate one that would obtain from a standard GL formula. All these spark renewed interest to two-gap superconductivity.

Principally, the two-gap superconductivity can be investigated in the frame of a charged two-condensate Bose system [8]. This system is described by a Ginzburg-Landau (GL) model with two flavors of Cooper pairs. Alternatively, it relates to a Gross-Pitaevskii (GP) functional with two charged condensates of tightly bound fermion pairs, or some other charged bosonic fields [9]. Such theoretical models have a wider range of applications, including interference between two Bose condensates [10], a multiband superconductor [11], two-component Bose-Einstein condensates [12] and superconducting gap structure of spintriplet superconductor $\mathrm{Sr}_{2} \mathrm{RuO}_{4}[13]$.

In this paper we mainly focus attention on the charged two-condensate Bose system. Since topological properties have played an important roles in the condensed matter, we shall be particularly interested in those of the charged two-condensate Bose system. We know that in

a single-condensate system, the best known topological object is the Abrikosov vortex [14]. But the advent of the charged two-condensate Bose system has opened a new possibility for us to have far more topological objects. Based on an extended version of Faddeev's $O(3)$ nonlinear $\sigma$ model, Babaev et. al. argued that the knotted soliton, which is a stable, finite-length, closed vortex line, exists in the charged two-condensate Bose system. The 
knotted solitons are much more complex and structurally complicated topological defects than Abrikosov vortices, and thus its realization should open an exceptionally wide range of possibilities of studies of various phenomena associated with them [15]. Generally, the numerical simulations method was adopted to research solitons [16], but to our knowledge there have been no real attempts to find an actual solution. Nevertheless the $\phi$-mapping method [17] provides a much briefer method, by making use of which one needs not to solve the nonlinear equation but directly investigates the knotted soliton from the point of view of topology. In this paper, using the novel topological current method, we study the topological properties of the charged two-condensate Bose system in more detail and naturally obtain two classes of knotted solitons, which are described by a nontrivial Hopf invariant and the $\mathrm{BF}$ action, respectively.

\section{TOPOLOGICAL VORTEX LINES IN THE TWO CHARGED SYSTEM}

A system with two electromagnetically coupled, oppositely charged Bose condensates can be described by a two-flavor (denoted by $\alpha=1,2$ ) Ginzburg-Landau or Gross-Pitaevskii (GLGP) functional, whose free energy density is given by

$$
\begin{aligned}
f= & \frac{1}{2 m_{1}}\left|\left(\hbar \partial_{\lambda}+i \frac{2 e}{c} A_{\lambda}\right) \Psi_{1}\right|^{2}+\frac{1}{2 m_{2}}\left|\left(\hbar \partial_{\lambda}-i \frac{2 e}{c} A_{\lambda}\right) \Psi_{2}\right|^{2} \\
& +V\left(\Psi_{1,2}\right)+\frac{\mathbf{B}^{2}}{8 \pi}
\end{aligned}
$$

where $V\left(\Psi_{1,2}\right)$ is expressed as: $V\left(\Psi_{1,2}\right)=-b_{\alpha}\left|\Psi_{\alpha}\right|^{2}+\frac{c_{\alpha}}{2}\left|\Psi_{\alpha}\right|^{4}$. The two condensates are characterized by the different effective masses $m_{\alpha}$, the different coherence lengths $\xi_{\alpha}=\hbar / \sqrt{2 m_{\alpha} b_{\alpha}}$ and the different concentrations $N_{\alpha}=\left\langle\left|\Psi_{\alpha}\right|^{2}\right\rangle=b_{\alpha} / c_{\alpha}$. The properties of the corresponding model with a single charged Bose field are well known. In that case the field degrees of freedom are the massive modulus of the single complex order parameter and a vector field which gains a mass due to the Meissner-Higgs effect. What is much important in the present GLGP model is that the two charged fields are not independent but nontrivially coupled through the electromagnetic field. This kind of nontrivial coupling indicates that in this system there should be a nontrivial, hidden topology which, however, cannot be recognized obviously in the form of Eq. (1). In order to find out the topological structure and to investigate it conveniently, Ref. [9] introduce a set of new variables, involving a massive field $\rho$ which is related to the densities of the Cooper pair and a three-component unit vector field 
$\hat{n}$. Then the original GLGP free energy density Eq. (1) can be represented as

$$
\begin{aligned}
f= & \frac{\hbar^{2} \rho^{2}}{4}(\partial n)^{2}+\hbar^{2}(\partial \rho)^{2}+\frac{\hbar^{2} c^{2}}{512 \pi e^{2}}\left\{\frac{1}{\hbar}\left[\partial_{\mu} C_{\nu}-\partial_{\nu} C_{\mu}\right]\right. \\
& \left.-\hat{n} \cdot \partial_{\mu} \hat{n} \times \partial_{\nu} \hat{n}\right\}^{2}+\frac{\rho^{2}}{16} \vec{C}^{2}+V .
\end{aligned}
$$

where $\vec{C}=\vec{J} / e \rho^{2}$ and $\vec{J}$ stands for the standard supercurrent. Now we can see that there allows an exact equivalence between the two-flavor GLGP model and the nonlinear $O(3)$ $\sigma$ model [16] which is much more important to describe the topological structure in high energy physics. In this paper, based on the decomposition of $U(1)$ gauge potential theory and the $\phi$-mapping method, we discuss the counterpart in condensed matter and show that there are topological excitations in the form of stable knotted closed vortices in the charged two-condensate Bose system. As shown in Eq. (2), we know that the magnetic field of the system can be divided into two parts: One is the contribution of field $C_{\mu}$, we learn that this part is introduced by the supercurrent density and can only present us with the topological defects as what in a single-condensate system; The other part, the contribution $\hat{n} \cdot \partial_{\mu} \hat{n} \times \partial_{\nu} \hat{n}$ to the magnetic field term in Eq. (2), is a fundamentally topological property of the two-condensate system which has no counterpart in the single-condensate system. Here we emphasize that there allow another nontrivial topological configurations, which are originated from the interaction between the field $C_{\mu}$ and the contribution of the term $\hat{n} \cdot \partial_{\mu} \hat{n} \times \partial_{\nu} \hat{n}$. Thus we will investigate these terms in detail.

It is easy to prove that this term $\hat{n} \cdot \partial_{\mu} \hat{n} \times \partial_{\nu} \hat{n}$ can be reexpressed in an Abelian field tensor form [19]: $\hat{n} \cdot \partial_{\mu} \hat{n} \times \partial_{\nu} \hat{n}=\partial_{\mu} b_{\nu}-\partial_{\nu} b_{\mu}$. Here $b_{\mu}$ is the Wu-Yang potential [20]

$$
b_{\mu}=\hat{e}_{1} \cdot \partial_{\mu} \hat{e}_{2}
$$

in which $\hat{e_{1}}$ and $\hat{e_{2}}$ are two perpendicular unit vectors normal to $\hat{n}$, and $\left(\hat{e}_{1}, \hat{e}_{2}, \hat{n}\right)$ forms an orthogonal frame: $\hat{n}=\hat{e_{1}} \times \hat{e_{2}}, \quad \hat{e}_{1} \cdot \hat{e}_{2}=0$. Now, consider a two-component vector field $\vec{\phi}=\left(\phi^{1}, \phi^{2}\right)$ residing in the plane formed by $\hat{e}_{1}$ and $\hat{e}_{2}: e_{1}^{a}=\frac{\phi^{a}}{\|\phi\|}, e_{2}^{a}=\epsilon_{a b} \frac{\phi^{b}}{\|\phi\|}, \quad\left(\|\phi\|^{2}=\right.$ $\left.\phi^{a} \phi^{a} ; a, b=1,2\right)$. It can be proved that the relations for $\hat{e}_{1}$ and $\hat{e}_{2}$ satisfy above restriction. Obviously the zero points of $\vec{\phi}=\left(\phi^{1}, \phi^{2}\right)$ are the singular points of $\hat{e}_{1}$ and $\hat{e}_{2}$. We will find out that the topological defects are just originated from these points. Using the $\vec{\phi}$ field, the Wu-Yang potential Eq. (3) can be expressed as $b_{\mu}=\epsilon_{a b} \frac{\phi^{a}}{\|\phi\|} \partial_{\mu} \frac{\phi^{b}}{\|\phi\|}$. Comparing with the expression of the $U(1)$ gauge potential decomposition [21], we learn that $b_{\mu}$ satisfies the $U(1)$ 
gauge transformation. The GLGP free energy density Eq. (2) can be reexpressed as

$$
\begin{aligned}
f= & \frac{\hbar^{2} \rho^{2}}{4}(\partial n)^{2}+\hbar^{2}(\partial \rho)^{2}+\frac{\hbar^{2} c^{2}}{512 \pi e^{2}} \\
& \times\left\{\frac{1}{\hbar}\left[\partial_{\mu} C_{\nu}-\partial_{\nu} C_{\mu}\right]-H_{\mu \nu}\right\}^{2}+\frac{\rho^{2}}{16} \vec{C}^{2}+V,
\end{aligned}
$$

where $H_{\mu \nu}$ is a two order tensor and can be expressed as: $H_{\mu \nu}=2 \epsilon_{a b} \partial_{\mu}\left(\phi^{a} /\|\phi\|\right) \partial_{\nu}\left(\phi^{b} /\|\phi\|\right)$, which describes the magnetic field that becomes induced in the system due to a nontrivial electromagnetic interaction between the two condensates. Indeed, it is exactly due to the presence of this term that the two-condensate system acquires properties which are qualitatively very different from those of a single-condensate system.

Since this tensor $H_{\mu \nu}$ in Eq. (4) plays an important role in the topological feature of the two-condensate system, here we will use the $\phi$-mapping method to research the properties hidden in this tensor. To do so, we introduce a topological tensor current $K^{\mu \nu}$ [21], which is denoted by

$$
K^{\mu \nu}=\frac{1}{8 \pi} \epsilon^{\mu \nu \lambda \rho} H_{\lambda \rho}=\frac{1}{4 \pi} \epsilon^{\mu \nu \lambda \rho} \epsilon_{a b} \partial_{\lambda} \frac{\phi^{a}}{\|\phi\|} \partial_{\rho} \frac{\phi^{b}}{\|\phi\|} .
$$

Using $\partial_{\mu}\left(\phi^{a} /\|\phi\|\right)=\left(\partial_{\mu} \phi^{a}\right) /\|\phi\|+\phi^{a} \partial_{\mu}(1 /\|\phi\|)$ and the Green function relation in $\phi$ space: $\partial_{a} \partial_{a} \ln \|\phi\|=2 \pi \delta^{2}(\vec{\phi})\left(\partial_{a}=\partial / \partial \phi^{a}\right)$, one can prove that $K^{\mu \nu}=\delta^{2}(\vec{\phi}) D^{\mu \nu}(\phi / x)$, where $D^{\mu \nu}(\phi / x)=\frac{1}{2} \epsilon^{\mu \nu \lambda \rho} \epsilon_{a b} \partial_{\lambda} \phi^{a} \partial_{\rho} \phi^{b}$ is the Jacobian between the $\phi$ space and the Cartesian coordinates. Denoting the spacial components of $K^{\mu \nu}$ by $K^{i}$, we obtain

$$
K^{i}=K^{0 i}=\delta^{2}(\vec{\phi}) D^{i}\left(\frac{\phi}{x}\right) \quad(i=1,2,3),
$$

where $D^{i}\left(\frac{\phi}{x}\right)=D^{0 i}\left(\frac{\phi}{x}\right)$. The expression Eq. (66) provides an important conclusion: $K^{i}=$ 0 , if and only if $\vec{\phi} \neq 0 ; K^{i} \neq 0$, if and only if $\vec{\phi}=0$. So it is necessary to study the zero points of $\vec{\phi}$ to determine the nonzero solutions of $K^{i}$. The implicit function theory [22] shows that under the regular condition $D^{i}(\phi / x) \neq 0$, the general solutions of

$$
\phi^{1}\left(t, x^{1}, x^{2}, x^{3}\right)=0, \quad \phi^{2}\left(t, x^{1}, x^{2}, x^{3}\right)=0
$$

can be expressed as $\vec{x}=\vec{x}_{k}(s, t)$, which represent the world surfaces of $N$ moving isolated singular strings $L_{k}$ with string parameter $s(k=1,2 \cdots N)$. This indicates that in the charged two-condensate Bose system, there are vortex lines located at the zero points of the $\vec{\phi}$ field.

Then question are raised naturally: what is the topological charge of the vortex lines and how to obtain the inner topological structure of $K^{i}$. Now we will investigate the topological 
charges of the vortex lines and their quantization. In $\delta$-function theory [23], one can prove that in three-dimensional space

$$
\delta^{2}(\vec{\phi})=\sum_{k=1}^{N} \beta_{k} \int_{L_{k}} \frac{\delta^{3}\left(\vec{x}-\vec{x}_{k}(s)\right)}{\left|D\left(\frac{\phi}{u}\right)\right|_{\Sigma_{k}}} d s
$$

where $D(\phi / u)_{\Sigma_{k}}=\frac{1}{2} \epsilon^{\mu \nu} \epsilon_{m n}\left(\partial \phi^{m} / \partial u^{\mu}\right)\left(\partial \phi^{n} / \partial u^{\nu}\right)$ and $\Sigma_{k}$ is the $k$ th planar element transverse to $L_{k}$ with local coordinates $\left(u^{1}, u^{2}\right)$. The positive integer $\beta_{k}$ is the Hopf index of the $\phi$ mapping, which means that when $\vec{x}$ covers the neighborhood of the zero point $\vec{x}_{k}(s)$ once, the vector field $\vec{\phi}$ covers the corresponding region in $\phi$ space $\beta_{k}$ times. Meanwhile taking notice of the definition of the Jacobian, the direction vector of $L_{k}$ is given by

$$
\left.\frac{d x^{i}}{d s}\right|_{\vec{x}_{k}}=\left.\frac{D^{i}(\phi / x)}{D(\phi / u)}\right|_{\vec{x}_{k}} .
$$

Then from Eqs. (8) and (9), we obtain the inner structure of $K^{i}$

$$
K^{i}=\delta^{2}(\vec{\phi}) D^{i}\left(\frac{\phi}{x}\right)=\sum_{k=1}^{N} \beta_{k} \eta_{k} \int_{L_{k}} \frac{d x^{i}}{d s} \delta^{3}\left(\vec{x}-\vec{x}_{k}(s)\right) d s,
$$

in which $\eta_{k}$ is the Brouwer degree of the $\phi$-mapping, with $\eta_{k}=\operatorname{sgn} D(\phi / u)_{\Sigma_{k}}= \pm 1$. Hence the topological charge of the vortex line $L_{k}$ is $Q_{k}=\int_{\Sigma_{k}} K^{i} d \sigma_{i}=W_{k}$, where $W_{k}=\beta_{k} \eta_{k}$ is the winding number of $\vec{\phi}$ around $L_{k}$. Then we come to the conclusion: under the regular condition $D^{i}(\phi / x) \neq 0$ there exist vortex lines in the charged two-condensate Bose system, whose topological charges are just the winding numbers of the $\phi$-mapping.

\section{TOPOLOGICAL PROPERTIES OF KNOTTED SOLITONS}

In this section, we research stationary knots corresponding to the vortex lines in this two-condensate system. When going to a constant vector $\hat{n}(x) \rightarrow \hat{n}_{0}, \hat{n}(x)$ naturally defines a mapping: $R_{3} \sim S^{3} \rightarrow S^{2}[16]$. Such mappings fall into nontrivial homotopy classes $\pi_{3}\left(S^{2}\right) \approx Z$ and can be characterized by a topological invariant called the Hopf invariant [24, 25], an integral formula which counts the number of times the two-sphere $S^{2}$ is covered by the three-sphere $S^{3}$. From the above discussion, the Hopf invariant can be given in terms of $b_{\mu}$ and $H_{\mu \nu}$ by [16]

$$
I=\frac{1}{16 \pi^{2}} \int \epsilon^{i j k} b_{i} H_{j k} d^{3} x
$$


where $b_{i}$ and $H_{j k}$ are the spacial components of $b_{\mu}$ and $H_{\mu \nu}$, respectively. It can be seen that when these $N$ vortex lines are closed curves, i.e., a family of knots $\gamma_{k}(k=1, \cdots, N)$, considering Eq. (5) and substituting Eq. (10) into Eq. (11), we obtain

$$
I=\frac{1}{2 \pi} \sum_{k=1}^{N} W_{k} \oint_{\gamma_{k}} b_{i} d x^{i} .
$$

This is a very important expression. Consider the $U(1)$ gauge transformation of $b_{\mu}: b_{\mu}^{\prime}=$ $b_{\mu}+\partial_{i} \theta$, where $\theta \in \mathbb{R}$ is a phase factor denoting the $U(1)$ transformation. It is seen that the term $\partial_{i} \theta$ contributes noting to the integral $I$, hence the expression (12) is invariant under the $U(1)$ gauge transformation. Meanwhile we know that $I$ is independent of the metric $g_{\mu \nu}$. Therefore one can conclude that $I$ is a topological invariant for knotted vortex lines in the charged two-condensate Bose system. In the presence of the vector field $\hat{n}$, the two-condensate system allows this kind of knots labelled by the Hopf invariant.

Simultaneously, it is due to the interaction between the massive field $C_{\mu}$ and the tensor $H_{\mu \nu}$ that a new nontrivially topological configuration will be produced in the two-condensate system. One can see that $C=C_{\mu} d x^{\mu}$ is a one-form, which presents us with the properties as what in a single-condensate system, and the two-form $H=\frac{1}{2} H_{\mu \nu} d x^{\mu} \wedge d x^{\nu}$ describes the magnetic field that becomes induced to the interaction between the two condensate. In order to explore the new topological configuration according to the interaction between the one-form $C$ and the two-form $H$, we introduce the classical Abelian BF theory [26, 27]. In this three-dimensional charged two-condensate system, the abelian BF theory has the following action

$$
S=\frac{1}{4 \pi} \int_{M} C \wedge H=\frac{1}{8 \pi} \int_{M} \epsilon^{i j k} C_{i} H_{j k} d x^{3},
$$

where $C_{i}$ and $H_{j k}$ are the spacial components of $C_{\mu}$ and $H_{\mu \nu}$. Considering the relation $K^{i}=\frac{1}{8 \pi} \epsilon^{i j k} H_{j k}$, one knows that there also allow the vortex lines. When the vortex lines are a family of knots, substituting Eq. (10) into Eq. (13), one can obtain

$$
S=\sum_{f=1}^{N} W_{f} \oint_{\gamma_{f}} C_{i} d x^{i} .
$$

We learn that $C_{\mu}$ satisfies the $U(1)$ gauge transformation: $C_{\mu}^{\prime}=C_{\mu}+\partial_{\mu} \varphi$ and $\varphi \in \mathbb{R}$ is a phase factor denoting the $U(1)$ transformation. Furthermore the term $\partial_{\mu} \varphi$ contributes noting to the integral $S$, hence the expression (14) is invariant under the $U(1)$ gauge transformation. Meanwhile one knows that $S$ is independent of the metric $g_{\mu \nu}$. Therefore we can conclude 
that the BF action $S$ is also a topological invariant for knotted vortex lines in the charged two-condensate Bose system.

It is well known that many important topological numbers are related to a knot family such as the self-linking number and Gauss linking number. Next we will discuss the relationship between the actions Eqs. (12]14) and the topological numbers of the knot family. First $b_{i}$ and $C_{i}$ should be expressed in terms of the vector field which carries the geometric information of the knot family. Define the Gauss mapping $\vec{m}: S^{1} \times S^{1} \rightarrow S^{2}$ by $\vec{m}(\vec{y}, \vec{x})=\frac{\vec{y}-\vec{x}}{\|\vec{y}-\vec{x}\|}$, where $\vec{x}$ and $\vec{y}$ are two points, respectively, on the knots $\gamma_{k}$ and $\gamma_{l}$. Let a two-dimensional unit vector $\vec{u}=\vec{u}(\vec{x}, \vec{y})$ satisfy $u^{a} u^{a}=1$, where $a=1,2$ and $\vec{u} \perp \vec{m}$. Then $b_{i}$ can be decomposed in terms of this two-dimensional vector $u^{a}: b_{i}=\epsilon^{a b} u^{a} \partial_{i} u^{b}-\partial_{i} \alpha$, in which $\alpha$ is a phase factor. it is due to the term $\partial_{i} \alpha$ contributes nothing to the integral Eq. (12) that $b_{i}$ can be written as $b_{i}=\epsilon^{a b} u^{a} \partial_{i} u^{b}$. And noticing the symmetry between the point $\vec{x}$ and $\vec{y}$, Eq. (12) can be reformulated as

$$
\begin{aligned}
I & =\frac{1}{2 \pi} \sum_{k=1}^{N} \sum_{l=1}^{N} W_{k} W_{l} \\
& \times \oint_{\gamma_{k}} \oint_{\gamma_{l}} \epsilon_{a b} \partial_{i} u^{a}(\vec{x}, \vec{y}) \partial_{j} u^{b}(\vec{x}, \vec{y}) d x^{i} \wedge d y^{j} .
\end{aligned}
$$

Further, we express the abelian BF action $S$ as a new form including a unit vector, which also carries geometric information. Introduce another Gauss mapping $\vec{d}: S^{1} \times S^{1} \rightarrow S^{2}$ and a two-dimensional unit vector field $\vec{v}\left(\overrightarrow{x^{\prime}}, \overrightarrow{y^{\prime}}\right)$, where $\overrightarrow{x^{\prime}}, \overrightarrow{y^{\prime}}$ are different points on $\gamma_{f}, \gamma_{g}$, and let $\vec{v}, \vec{d}$ satisfy $\vec{v} \perp \vec{d}$. Then using the $U(1)$ gauge potential decomposition theory, one can obtain that $C_{i}$ is decomposed in terms of the vector $\vec{v}: C_{i}=\epsilon^{a b} v^{a} \partial_{i} v^{b}+\partial_{i} \beta$, where $\beta$ is a phase factor and $\partial_{i} \beta$ has no contribution to the integral Eq. (14). Substituting the expression of the decomposition into the BF action Eq. (14) and taking notice of the symmetry between the point $\overrightarrow{x^{\prime}}$ and $\overrightarrow{y^{\prime}}$, we get

$$
\begin{aligned}
S & =\sum_{f=1}^{N} \sum_{g=1}^{N} W_{f} W_{g} \\
& \times \oint_{\gamma_{f}} \oint_{\gamma_{g}} \epsilon_{a b} \partial_{i} v^{a}\left(\overrightarrow{x^{\prime}}, \overrightarrow{y^{\prime}}\right) \partial_{j} v^{b}\left(\overrightarrow{x^{\prime}}, \overrightarrow{y^{\prime}}\right) d x^{i} \wedge d y^{\prime j}
\end{aligned}
$$

In fact there exist three cases for Hopf invariant Eq. (15) and the BF action Eq. (16), respectively. (i) The same knots but the different points, i.e., $k=l, \vec{x} \neq \vec{y}$ or $f=g, \overrightarrow{x^{\prime}} \neq \overrightarrow{y^{\prime}}$; (ii) The same knots and the same points, i.e., $k=l, \vec{x}=\vec{y}$ or $f=g, \overrightarrow{x^{\prime}}=\overrightarrow{y^{\prime}}$; (iii) The 
different knots, i.e., $k \neq l$ or $f \neq g$. For the first case, when $\gamma_{k}, \gamma_{l}$ are the same knots, $\gamma_{f}, \gamma_{g}$ are the same knots, but $\vec{x}$ and $\vec{y}, \overrightarrow{x^{\prime}}$ and $\overrightarrow{y^{\prime}}$ are different points, taking account of the relations $\epsilon_{a b} \partial_{i} u^{a} \partial_{j} u^{b}=\frac{1}{2} \vec{m} \cdot\left(\partial_{i} \vec{m} \times \partial_{j} \vec{m}\right)$ and $\epsilon_{a b} \partial_{i} v^{a} \partial_{j} v^{b}=\frac{1}{2} \vec{d} \cdot\left(\partial_{i} \vec{d} \times \partial_{j} \vec{d}\right)$, one can prove that

$$
\begin{aligned}
& \frac{1}{2 \pi} \oint_{\gamma_{k}} \oint_{\gamma_{k}} \epsilon_{a b} \partial_{i} u^{a} \partial_{j} u^{b} d x^{i} \wedge d y^{j}=\frac{1}{4 \pi} \oint_{\gamma_{k}} \oint_{\gamma_{k}} \vec{m}^{*}(d S) \\
& \frac{1}{2 \pi} \oint_{\gamma_{f}} \oint_{\gamma_{f}} \epsilon_{a b} \partial_{i} v^{a} \partial_{j} v^{b} d x^{i} \wedge d y^{\prime j}=\frac{1}{4 \pi} \oint_{\gamma_{f}} \oint_{\gamma_{f}} \vec{d}^{*}(d S)
\end{aligned}
$$

where $\vec{m}^{*}(d S)=\vec{m} \cdot(d \vec{m} \times d \vec{m})=2 \epsilon_{a b} d u^{a} \wedge d u^{b}$ is the pull-back of $S^{2}$ surface element. The expressions is just related to the writhing number $W r\left(\gamma_{k}\right)$ of $\gamma_{k}: W\left(\gamma_{k}\right)=\frac{1}{4 \pi} \oint_{\gamma_{k}} \oint_{\gamma_{k}} \vec{m}^{*}(d S)$. For the second case, $\gamma_{k}, \gamma_{l}$ are the same knots, $\gamma_{f}, \gamma_{g}$ are the same, $\vec{x}$ and $\vec{y}$ are just the same points, $\overrightarrow{x^{\prime}}$ and $\overrightarrow{y^{\prime}}$ are also the same. Let $\vec{T}\left(\overrightarrow{T^{\prime}}\right)$ be the unit tangent vector of knot $\gamma_{k}\left(\gamma_{f}\right)$ at $\vec{x}\left(\overrightarrow{x^{\prime}}\right)$ and let $\vec{V}\left(\vec{V}^{\prime}\right)$ satisfy $u^{a}=\epsilon^{a b} V^{b}\left(v^{a}=\epsilon^{a b} V^{\prime b}\right)$, where $a, b=1,2 ; \vec{V} \perp \vec{T}, \vec{u}=\vec{T} \times \vec{V}$ $\left(\overrightarrow{V^{\prime}} \perp \overrightarrow{T^{\prime}}, \vec{v}=\vec{T}^{\prime} \times \vec{V}^{\prime}\right)$, then it is easy to prove

$$
\begin{aligned}
& \frac{1}{2 \pi} \oint_{\gamma_{k}} \epsilon_{a b} u^{a} \partial_{i} u^{b} d x^{i}=\frac{1}{2 \pi} \oint_{\gamma_{k}}(\vec{T} \times \vec{V}) \cdot d \vec{V}=T w\left(\gamma_{k}\right), \\
& \frac{1}{2 \pi} \oint_{\gamma_{f}} \epsilon_{a b} v^{a} \partial_{i} v^{b} d x^{i}=\frac{1}{2 \pi} \oint_{\gamma_{f}}\left(\vec{T}^{\prime} \times \vec{V}^{\prime}\right) \cdot d \vec{V}^{\prime}=T w\left(\gamma_{f}\right),
\end{aligned}
$$

where $T w\left(\gamma_{k}\right)$ and $T w\left(\gamma_{f}\right)$ are the twisting numbers of the knot $\gamma_{k}$ and $\gamma_{f}$. According to the Calugareanu's formula [28, 29]: $S L\left(\gamma_{k}\right)=W r\left(\gamma_{k}\right)+T w\left(\gamma_{k}\right)$, where $S L\left(\gamma_{k}\right)$ is just the self-linking number of $\gamma_{k}$, one can arrive at the result that when $\gamma_{k}$ and $\gamma_{l}, \gamma_{f}$ and $\gamma_{g}$ are the same knots i.e. $k=l, f=g$, the actions Eqs. (15) and (16) are related to the self-linking of knots. For the third case, when $\gamma_{k}$ and $\gamma_{l}, \gamma_{f}$ and $\gamma_{g}$ are different knots, considering the relations between $\vec{u}$ and $\vec{m}, \vec{v}$ and $\vec{d}$, we can obtain

$$
\begin{aligned}
\frac{1}{2 \pi} \oint_{\gamma_{k}} \oint_{\gamma_{l}} \epsilon_{a b} \partial_{i} u^{a} \partial_{j} u^{b} d x^{i} \wedge d y^{j} & =\frac{1}{4 \pi} \oint_{\gamma_{k}} \oint_{\gamma_{l}} \vec{m}^{*}(d S) \\
\frac{1}{2 \pi} \oint_{\gamma_{f}} \oint_{\gamma_{g}} \epsilon_{a b} \partial_{i} v^{a} \partial_{j} v^{b} d x^{i} \wedge d y^{\prime j} & =\frac{1}{4 \pi} \oint_{\gamma_{f}} \oint_{\gamma_{g}} \vec{d}^{*}(d S),
\end{aligned}
$$

in which $\frac{1}{4 \pi} \oint_{\gamma_{k}} \oint_{\gamma_{l}} \vec{m}^{*}(d S)=\frac{1}{4 \pi} \epsilon^{i j k} \oint_{\gamma_{k}} d x^{i} \oint_{\gamma_{l}} d y^{i} \frac{\left(x^{k}-y^{k}\right)}{\|\vec{x}-\vec{y}\|^{3}}=L\left(\gamma_{k}, \gamma_{l}\right)$ is the Gauss linking number between $\gamma_{k}$ and $\gamma_{l}, \frac{1}{4 \pi} \oint_{\gamma_{f}} \oint_{\gamma_{g}} \overrightarrow{d^{*}}(d S)=L\left(\gamma_{f}, \gamma_{g}\right)$ is the Gauss linking number between $\gamma_{f}$ and 
$\gamma_{g}$. Therefore, taking account of all these three cases, we come to the important conclusions

$$
\begin{aligned}
I & =\sum_{k=1}^{N} \sum_{l=1}^{N} W_{k} W_{l} L\left(\gamma_{k}, \gamma_{l}\right)+\sum_{k=1}^{N} W_{k}^{2} S L\left(\gamma_{k}\right), \\
S & =2 \pi\left[\sum_{f=1}^{N} \sum_{g=1}^{N} W_{f} W_{g} L\left(\gamma_{f}, \gamma_{g}\right)+\sum_{f=1}^{N} W_{f}^{2} S L\left(\gamma_{f}\right)\right],
\end{aligned}
$$

which describe the twisted knot topology of the physical magnetic flux. Since the self-linking number and the Gauss linking number are both the invariant characteristic numbers of the knotted closed curves in topology, $I$ and $S$ are also important topological invariants required to describe the knotted vortex lines in the charged two-condensate system. Notice that they can be understood as the linking of two quantized magnetic fluxes of the knotted vortex lines. Obviously two flux rings linked together can not be separated by any continuous deformation of the field configuration. This provides the topological stability of the knots. Since the energy of a vortex line is proportional to its length, for a finite energy the length must be finite [16]. Then we obtain a stable, finite length closed vortex lines in the charged two-condensate Bose system, namely, knotted solitions. This is just the significance of the introduction and research of topological invariants $I$ and $S$.

\section{CONCLUSION}

In conclusion, based on the decomposition of $U(1)$ gauge potential theory and the $\phi$ mapping topological current method, we point out there allow vortex lines and two classes of knotted solitons in the charged two-condensate Bose system. Under the regular condition $D^{i}(\phi / x) \neq 0$, vortex lines are originated form the zero points of the $\vec{\phi}$ field and the topological charges of vortex lines are characterized by the winding numbers of the $\phi$-mapping. Furthermore, we discuss the stationary knots and find out two classes of knotted solitonsstable, finite length, closed vortex lines in the two-condensate system. One knotted solition is described by the Hopf invariant and the other is characterized by the BF action. Finally, the relations between these actions and the topological numbers of a family knot are investigated in the system. It is revealed that these actions are topological invariants for

knotted solitons and can be reformulated into the self-linking numbers and the Gauss linking numbers.

At last, it should be pointed out that in the present paper we treat the vortex lines as 
mathematical lines, i.e., the width of a line is zero. This description is obtained in the approximation that the radius of curvatures of a line is much larger than the width of the line [30].

\section{Acknowledgments}

It is a great pleasure to thank Dr. P. M. Zhang for numerous fruitful discussions. This

work was supported by the National Natural Science Foundation of China under Grant No. 10475034 .

[1] H. Suhl, B.T. Matthias, and L.R. Walker, Phys. Rev. Lett. 3, 552 (1959).

[2] V.A. Moskalenko, Fiz. Met. Metalloved. 8, 503 (1959).

[3] A.Y. Liu, I.I. Mazin, and J. Kortus, Phys. Rev. Lett. 87, 087005 (2001).

[4] H.J. Choi, D. Roundy, H. Sun, M.L. Cohen, and S.G. Louie, Phys. Rev. B 66, 020513 (2002).

[5] A. Floris, G. Profeta, N.N. Lathiotakis, M. Lüders, M.A.L. Marques, C. Franchini, E.K.U. Gross, A. Continenza, and S. Massidda, Phys. Rev. Lett. 94, 037004 (2005).

[6] V.H. Dao, and M.E. Zhitomirsky, Eur. Phys. J. B 44, 183 (2005).

[7] M.R. Eskildsen, M. Kugler, S. Tanaka, J. Jun, S.M. Kazakov, J. Karpinski, and Ø. Fischer, Phys. Rev. Lett. 89, 187003 (2002).

[8] Y. Jiang, Phys. Rev. B, 70, 012501 (2004).

[9] E. Babaev, L.D. Faddeev, and A.J. Niemi, Phys. Rev. B 65, 100512 (2002).

[10] M.R. Andrews, C.G. Townsend, H.J. Miesner, D.S. Durfee, D.M. Kurn, and W. Ketterle, Scince 275, 637 (1997).

[11] F. Bouquet, R.A. Fisher, N.E. Phillips, D.G. Hinks, and J.D. Jorgensen, Phys. Rev. Lett. 87, 047001 (2001); P. Szabó, P. Samuely, J. Kačmarčík, T. Klein, J. Marcus, D. Fruchart, S. Miraglia, C. Marcenat, and A.G. M. Jansen, Phys. Rev. Lett. 87, 137005 (2001).

[12] D.S. Hall, M.R. Matthews, C.E. Wieman, and E.A. Cornell, Phys. Rev. Lett. 81, 1543 (1998).

[13] K. Izawa, H. Takahashi, H. Yamaguchi, Y. Matsuda, M. Suzuki, T. Sasaki, T. Fukase, Y. Yoshida, R. Settai, and Y. Onuki, Phys. Rev. Lett. 86, 2653 (2001). 
[14] Y.M. Cho, and P.M. Zhang, Non-Abrikosov Vortex and Topological Knot in Two-gap Superconductor, cond-mat/0601347.

[15] E. Babaev, Phy. Rev. Lett. 88, 177002 (2002).

[16] L. Faddeev, and A.J. Niemi, Nature (London) 387, 58 (1997); Phys. Rev. Lett. 82, 1624 (1999).

[17] Y.S. Duan, S. Li, and G.H. Yang, Nucl. Phys. B 514, 705 (1998); Y. Jiang and Y.S. Duan, J. Math. Phys. 24, 6463 (2000).

[18] A.J. Leggett, Prog. Theor. Phys. 36, 901 (1996); E. Babaev, Nucl. Phys. B 686, 397 (2004).

[19] F. Wilczek, and A. Zee, Phys. Rev. Lett. 51, 2250 (1983).

[20] T.T. Wu, and C.N. Yang, Phys. Rev. D 12, 3845 (1975); 14, 437 (1975).

[21] Y.S. Duan, X. Liu, and P.M. Zhang, J. Phys. A 36, 563 (2003); Y.S. Duan, H. Zhang, and S. Li, Phys. Rev. B 58, 125 (1998).

[22] É. Goursat, A Course in Mathematical Analysis, translated by E.R. Hedrick (Dover, New York, 1904), Vol. I.

[23] J.A. Schouten, Tensor Analysis for Physicists (Clarendon, Oxfors, 1951).

[24] L.H. Kauffman, Knots and Physics (World Scientific, Singapore, 1993).

[25] M. Atiyah, The Geometry and Physics of Knots (Cambridge Univ. press, 1990).

[26] A. Borowiec and M. Francaviglia, AIP Conf. Proc. 751, 165 (2005); A. Momen, Physics Letters B 394, 269 (1997).

[27] N. Ikeda, JHEP 0011, 009 (2000); JHEP 0107, 037 (2001).

[28] D. Rolfsen, Knots and Links (Publish or Perish, Berkeley, CA, 1976).

[29] W. Pohl, J. Math. Mech. 17, 975 (1968).

[30] H.B. Nielsen and P. Olesen, Nucl. Phys. B 61, 45 (1973). 